\title{
ON THE CURVATURES OF A CURVE IN RIEMANN SPACE* ${ }^{*}$
}

\author{
BY \\ E. H. CUTLER
}

Introduction. The curvature and torsion of a curve in ordinary space have three properties which it is the purpose of this paper to attempt to extend to the curvatures of a curve in Riemann space. First, if the curvature vanishes identically the curve is a straight line; if the torsion vanishes identically the curve lies in a plane. Second, the distances of a point of the curve from the tangent line and the osculating plane at a nearby point are given approximately by formulas involving the curvature and torsion. Third, the curvature of a curve at a point is the curvature of its projection on the osculating plane at the point. In extending to Riemann space we take as the Riemannian analogue of the line or plane, a geodesic space generated by geodesics through a point. Such a space possesses the property of the line or plane of being determined by the proper number of directions given at a point, but it will not in general have the three properties given above. On the other hand, if we take as the analogue of line or plane only totally geodesic spaces, then, if such osculating "planes" exist, the three properties will hold.

Curves with a vanishing curvature. Given a curve $C: x^{i}=x^{i}(s), i=1$, $\cdots, n$, in a Riemann space $V_{n}$ with fundamental tensor $g_{i j}$ (assumed definite). Following Blaschke $\ddagger$ we write the Frenet formulas for the curve. The $n$ associate vectors are given by

$$
\left.\xi_{1}\right|^{i}=\frac{d x^{i}}{d s},\left.\quad \xi_{r}\right|^{i}, i \frac{d x^{j}}{d s}=\left.\xi_{r+1}^{\prime}\right|^{i}(r=1, \cdots,(n-1)) .
$$

In general these $n$ vectors are independent and will determine an orthogonal $n$-uple, $\left.\lambda_{r}\right|^{i}$, in terms of which we have the Frenet formulas

$$
\left.\lambda_{1}\right|^{i}=\left.\xi_{1}\right|^{i}=\frac{d x^{i}}{d s}
$$

$$
\left.\lambda_{r}\right|^{i}, j \frac{d x^{j}}{d s}=-\left.\left(1 / \rho_{r-1}\right) \lambda_{r-1}\right|^{i}+\left.\left(1 / \rho_{r}\right) \lambda_{r+1}\right|^{i}, \quad r=1, \cdots, n,\left(1 / \rho_{n}\right)=0 .
$$

* Presented to the Society, February 28, 1931; received by the editors February 10, 1931.

$\uparrow$ The results of this and the following paper are part of a thesis submitted at Harvard, June 1930, and written under Professor H. W. Brinkmann.

$\ddagger$ Blaschke, Frenets Formeln für den Raum von Riemann, Mathematische Zeitschrift, vol. 6, pp. 94-99. See also Eisenhart, Riemannian Geometry, \$32. 
If, however, there are, at a general point of the curve, only $k(k<n)$ independent associate vectors, then (2) will hold for $r=1, \ldots, k$ where $\left(1 / \rho_{k}\right)=0$. This case will be described by saying $\left(1 / \rho_{k}\right)=0$. In the general case the vector $\left.\xi_{r}\right|^{i}$ is always dependent on $\left.\lambda_{1}\right|^{i}, \ldots,\left.\lambda_{r}\right|^{i}$, and in this case the associate vectors of all orders will be dependent on $\lambda_{1}\left|{ }^{i}, \cdots, \lambda_{k}\right|{ }^{i}$.

Definition. The linear vector space at $P$ determined by the vectors $\lambda_{r} \mid i$, $r=1, \cdots, q$, will be the qth osculating vector space of $C$ at $P$. If $\left(1 / \rho_{k}\right)=0$, the kth osculating vector space will be called the complete osculating vector space.

We wish to discuss the relations of $C$ to its complete osculating geodesic space $G_{k}$ at $P$, where $G_{k}$ is the locus of the $\infty^{k-1}$ geodesics through $P$ in directions of the complete osculating vector space. To discuss the question we take Riemannian coördinates at $P$. Then $G_{k}$ is given by linear equations, and we wish to see whether the vector $\left[x^{i}(s)-x^{i}(P)\right]$ satisfies these, or, in other words, whether in this coördinate system it is linearly dependent on $\left.\lambda_{1}\right|^{i}$, $\cdots,\left.\lambda_{k}\right|^{i}$. Expanding $x^{i}(s)$ about $P$, the question reduces to that of the dependence of the ordinary derivatives of $x^{i}(s)$ of all orders on the $\lambda_{q} \mid{ }^{i}, q=1$, $\cdots, k$, or on the $\xi_{r} \mid i, r=1,2, \cdots$, since these are so dependent. From (1) we have

$$
\begin{aligned}
\left.\xi_{1}\right|^{i}= & \left.\lambda_{1}\right|^{i}=\frac{d x^{i}}{d s}, \\
\left.\xi_{2}\right|^{i}= & \frac{d^{2} x^{i}}{d s^{2}}+\left\{\begin{array}{c}
i \\
j k
\end{array}\right\} \frac{d x^{i}}{d s} \frac{d x^{k}}{d s} \\
\left.\xi_{3}\right|^{i}= & \frac{d^{3} x^{i}}{d s^{3}}+3\left\{\begin{array}{c}
i \\
j k
\end{array}\right\} \frac{d^{2} x^{i}}{d s^{2}} \frac{d x^{k}}{d s}+\left[\frac{\partial}{\partial x^{h}}\left\{\begin{array}{c}
i \\
j k
\end{array}\right\}+\left\{\begin{array}{c}
i \\
j l
\end{array}\right\}\left\{\begin{array}{c}
l \\
k h
\end{array}\right\}\right] \frac{d x^{h}}{d s} \frac{d x^{j}}{d s} \frac{d x^{k}}{d s} \\
\left.\xi_{4}\right|^{i}= & \frac{d^{4} x^{i}}{d s^{4}}+5 \frac{\partial}{\partial x^{h}}\left\{\begin{array}{c}
i \\
j k
\end{array}\right\} \frac{d^{2} x^{i}}{d s^{2}} \frac{d x^{k}}{d s} \frac{d x^{h}}{d s}+\frac{\partial}{\partial x^{h}}\left\{\begin{array}{c}
i \\
j k
\end{array}\right\} \frac{d x^{j}}{d s} \frac{d x^{k}}{d s} \frac{d^{2} x^{h}}{d s^{2}} \\
& +\frac{\partial^{2}}{\partial x^{h} \partial x^{i}}\left\{\begin{array}{c}
i \\
j k
\end{array}\right\} \frac{d x^{h}}{d s} \cdots \frac{d x^{k}}{d s}+\text { terms in undifferentiated }\left\{\begin{array}{c}
i \\
j k
\end{array}\right\} .
\end{aligned}
$$

The equations (3) hold in any coördinate system. For a Riemannian system of coördinates we have, at the center $P$,

$$
\begin{aligned}
\left\{\begin{array}{c}
i \\
j k
\end{array}\right\} & =0, \\
S\left(\frac{\partial^{u}}{\partial x^{h} \cdots \partial x^{l}}\left\{\begin{array}{c}
i \\
j k
\end{array}\right\}\right) & =0, \quad S=\text { symmetric part } .
\end{aligned}
$$


From (3) and (4) it follows that the first three derivatives of $x^{i}(s)$ at $P$ are actually equal to the corresponding $\left.\xi_{r}\right|^{i}$, and that for $\left.\xi_{4}\right|^{i}$ we will have at $P$

$$
\left.\xi_{4}\right|^{i}=\frac{d^{4} x^{i}}{d s^{4}}+3 \frac{\partial}{\partial x^{h}}\left\{\begin{array}{c}
i \\
j k
\end{array}\right\} \frac{d^{2} x^{j}}{d s^{2}} \frac{d x^{h}}{d s} \frac{d x^{k}}{d s} .
$$

Using (4) and the definition of $R_{j k l}^{i}$, we show that at $P$

$$
\begin{aligned}
3 \frac{\partial}{\partial x^{h}}\left\{\begin{array}{c}
i \\
j l
\end{array}\right\} & =R^{i}{ }_{j h l}-R^{i}{ }_{l j h}, \\
\left.\xi_{4}\right|^{i} & =\frac{d^{4} x^{i}}{d s^{4}}-R^{i}{ }_{j l h} \frac{d^{2} x^{l}}{d s^{2}} \frac{d x^{i}}{d s} \frac{d x^{h}}{d s} .
\end{aligned}
$$

The second term in general neither vanishes nor lies in any special vector space, as may be shown by examples. Hence the theorem we were seeking does not hold in general. A special case for which it does hold is that for which the $G_{k}$ is totally geodesic in $V_{n}$. To show this we make use of a development for $\left(\partial^{r} / \partial x^{l} \cdots \partial x^{h}\right)\left\{j_{j k}^{i}\right\}$, due to Veblen, ${ }^{*}$ of which (5) is a special case. According to this, $\left(\partial^{r} / \partial x^{l} \cdots \partial x^{h}\right)\left\{\begin{array}{l}i \\ j\end{array}\right\}$ is a combination of terms in $R^{i}{ }_{j k l}, \ldots h$, subscripts permuted in all possible ways, and of terms of inner products of similar factors of lower orders. Beside this we need a lemma.

Lemma. Let $G_{k}$ be totally geodesic in $V_{n}$ and let $\left.\eta_{1}\right|^{i} \cdots \eta_{s} \mid{ }^{i}$ be any vectors tangent to $G_{k}$. Then the vectors $\left.R^{i}{ }_{j k l} \eta_{1}\left|{ }^{i} \eta_{2}\right|{ }^{k} \eta_{3}\left|{ }^{l}, \ldots, R^{i}{ }_{j k l}, \ldots{ }_{h} \lambda_{1}\right|^{i} \cdots \lambda_{s}\right|^{h}$ lie in $G_{k}$.

To prove this we recall that for a totally geodesic $G_{k}: x^{i}=x^{i}\left(u^{1}, \cdots, u^{k}\right)$,

$$
\left.\Omega_{\sigma}\right|_{\alpha \beta} \equiv 0 \quad(\alpha, \beta=1, \cdots, k ; \sigma=1, \cdots,(n-k)),
$$

and hence by the Codazzi equation

$$
R_{i j k l \xi_{\sigma}} \mid i \frac{\partial x^{j}}{\partial u^{\alpha}} \frac{\partial x^{k}}{\partial u^{\beta}} \frac{\partial x^{l}}{\partial u^{\gamma}}=0 \quad(\alpha, \beta, \gamma=1, \cdots, k),
$$

or $R^{i}{ }_{j k l} \eta_{1}\left|{ }^{i} \eta_{2}\right|{ }^{k} \eta_{3} \mid{ }^{l}$ lies in $G_{k}$. Differentiating (7) covariantly, and using (6) and (7), we have

$$
R_{i j k l, h} \xi_{o} \mid i \frac{\partial x^{i}}{\partial u^{\alpha}} \frac{\partial x^{k}}{\partial u^{\beta}} \frac{\partial x^{l}}{\partial u^{\gamma}} \frac{\partial x^{h}}{\partial u^{\delta}}=0,
$$

and similarly for continued differentiation. Hence the lemma.

Combining this lemma, which holds for any coördinate system, with the expression for the derivatives of the $\left\{\begin{array}{c}i \\ j k\end{array}\right\}$ at the center of a Riemannian coördinate system in terms of the $R_{j k l}^{i}$ and its derivatives, we have

* O. Veblen, Proceedings of the National Academy of Sciences, vol. 8, p. 196. 
THEOREM 1. If $G_{k}$ is totally geodesic in $V_{n}$ and the coordinates are Riemannian at $P$, and if $\left.\eta_{1}\right|^{i}, \cdots,\left.\eta_{s}\right|^{i}$ lie in $G_{k}$, then

$$
\left.\left.\frac{\partial^{r}}{\partial x^{h} \cdots \partial x^{l}}\left\{\begin{array}{c}
i \\
j k
\end{array}\right\} \eta_{1}\right|^{h} \cdots \eta_{8}\right|^{k}
$$

is a vector in $G_{k}$.

Now assume that $G_{k}$, the complete osculating geodesic space of the curve $C$ at $P$, is totally geodesic in $V_{n}$. By (3) we see that the $r$ th derivative of $x^{i}(s)$ depends on $\left.\xi_{r}\right|^{i}$ and on terms of type (9), or products of factors of type (9) where the $\left.\eta_{s}\right|^{i}$ are derivatives of $x^{i}(s)$ of order $l, l<r$. Hence by the theorem above, and by induction, we show that the derivatives of $x^{i}(s)$ of all orders lie in $G_{k}$. Hence the curve lies in $G_{k}$.

THEOREM 2. If the kth curvature $(k<n)$ of a curve is identically zero, and if the complete osculating geodesic space $G_{k}$ at a point $P$ is totally geodesic, then the curve lies in $G_{k}$.

COROLLARY. If the kth curvature of a curve in a space of constant curvature vanishes identically, then the curve lies in the complete osculating geodesic space at any point.

Distance from an osculating geodesic space. Given a curve in $V_{n}$ which now is not assumed to have vanishing curvatures. We wish to obtain a formula for the distance of a point $P^{\prime}$ of the curve from the various osculating geodesic spaces $G_{h}$ at a nearby point $P$. Let $P$ be the center of a system of normal Riemannian coördinates so chosen that $G_{h}$ is given by

$$
x^{i}=0 \quad(i=(h+1), \cdots, n) .
$$

THEOREM 3. Given a system of normal Riemannian coördinates with center at $P:(0)$, and given a sequence of points $P_{r}:\left(x^{i}\right)$ approaching $P$ as a limit, then the principal part of the infinitesimal distance of $P_{r}$ from the geodesic sub-space $G_{h}: x^{i}=0, i=(h+1), \cdots, n, i$ given by

$$
d^{2}=\sum_{i=h+1}^{n}\left(x^{i}\right)^{2}+\cdots
$$

The proof of this consists first in showing that the true length of the geodesic from $P_{r}$ orthogonal to $G_{k}$ and the length this geodesic would have in terms of $\delta_{i j}$ as fundamental tensor are equivalent infinitesimals. The same is true for the lengths of the curves through $P_{r}$ which would be the orthogonal geodesics if $\delta_{i j}$ were fundamental tensor. From these facts and the fact that a geodesic gives the shortest distance the theorem follows. 
We now apply the theorem to find approximately the distance of a point $P^{\prime}$ of the curve from the osculating geodesic space at a nearby point $P$ by expanding $x^{i}(s)$ about $P$.

TheOReM 4. Given a system of normal Riemannian coördinates at $P:(0)$, then the principal part of the distance of a nearby point $P^{\prime}$ of $C$ from $G_{h}: x^{i}=0$, $i=(h+1), \cdots, n$, is given by

$$
d^{2}=\left[\frac{(\Delta s)^{u}}{u !}\right]^{2} \sum_{i=h+1}^{n}\left(\frac{d^{u} x^{i}}{d s^{u}}\right)^{2}+\cdots,
$$

where $u$ is the least number for which the uth derivative of $x^{i}(s)$ in this coördinate system does not lie in $G_{k}$.

This means that $d$ is approximately $(\Delta s)^{u} / u$ ! times length of component of $u$ th derivative normal to $G_{k}$. Taking $G_{h}$ as the tangent geodesic $(h=1)$, we have $u=2$ and $d^{2} x^{i} / d s^{2}=\left.\xi_{2}\right|^{i}$

$$
d=\frac{1}{2 \rho_{1}}(\Delta s)^{2}+\cdots .
$$

Equation (13) will hold in any coördinate system. Similarly, taking $G_{2}$ as determined by $\left.\lambda_{1}\right|^{i}$ and $\left.\lambda_{2}\right|^{i}$, we have $u=3$ and

$$
\begin{aligned}
& \frac{d^{3} x^{i}}{d s^{3}}=\left.\xi_{3}\right|^{i}+\text { vector in } G_{2}, \\
& \left.\xi_{3}\right|^{i}=-\left.\left(1 / \rho_{1}\right)^{2} \lambda_{1}\right|^{i}+\left.\frac{d}{d s}\left(1 / \rho_{1}\right) \lambda_{2}\right|^{i}+\left.\left(1 /\left(\rho_{1} \rho_{2}\right)\right) \lambda_{3}\right|^{i} .
\end{aligned}
$$

Hence the only component perpendicular to $G_{2}$ is $\left.\left(1 /\left(\rho_{1} \rho_{2}\right)\right) \lambda_{3}\right|^{i}$ and

$$
d=(\Delta s)^{3} /\left(6\left(\rho_{1} \rho_{2}\right)\right)+\cdots \cdot
$$

Higher than this we cannot go because of the impossibility of replacing the derivatives of $x^{i}(s)$ by the corresponding associate vectors. We note however that if $G_{3}$ is determined by $\left.\lambda_{1}\right|^{i},\left.\lambda_{2}\right|^{i},\left.\lambda_{3}\right|^{i}$ the distance of $P^{\prime}$ from $G_{3}$ is an infinitesimal of at least the fourth order.

Theorem 5. The principal parts of the infinitesimal distances of $P^{\prime}$ from the osculating $G_{1}$ and $G_{2}$ at $P$ are given by (13) and (14), and the distance from $G_{3}$ is an infinitesimal of at least the fourth order.

Now assume that $G_{h}$, the $h$ th osculating geodesic space of the curve at $P$, is totally geodesic. The vector $\left(d^{r} x^{i} / d s^{r}-\left.\xi_{r}\right|^{i}\right)$ consists of vectors $d^{l} x^{i} / d s^{l}, l<r$, contracted into partial derivatives of $\left\{\begin{array}{c}i \\ j k\end{array}\right\}$, and by Theorem 1 we show by steps that $d^{r} x^{i} / d s^{r}$ lies in $G_{h}$ whenever $\left.\xi_{r}\right|^{i}$ does; that is, for $r \leqq h$. Also the 
vector $\left(d^{h+1} x^{i} / d s^{h+1}-\xi_{h+1} \mid i\right)$ must lie in $G_{h}$ and the component of $d^{h+1} x^{i} / d s^{h+1}$ normal to $G_{h}$ is the same as that of $\xi_{h+1} \mid i$. But by (2) we have

$$
\left.\xi_{h+1}\right|^{i}=\text { vector in } G_{h}+\left.\left(1 /\left(\rho_{1} \rho_{2} \cdots \rho_{h}\right)\right) \lambda_{h+1}\right|^{i},
$$

and by Theorem 4,

$$
d=(\Delta s)^{h+1} /\left[(h+1) ! \rho_{1} \rho_{2} \cdots \rho_{h}\right]+\cdots .
$$

Theorem 6. If the kth osculating geodesic space at $P$ to $C$ is totally geodesic, then the principal part of the distance of a nearby point $P^{\prime}$ from it is given by (15).

Projection of curve on osculating space. Another property of ordinary space curves which we shall extend where possible to curves in a Riemann space is that certain curvatures of the curve $C$ are equal to the corresponding curvatures of the projection of $C$ on one of its osculating spaces. For the Riemannian case the osculating spaces are osculating geodesic spaces and projection is by means of the orthogonal geodesics.

As before we take normal Riemannian coördinates at $P$, so that the osculating space in question is given by $x^{i}=0, i>h, C$ is given by $x^{i}=x^{i}(s)$, $i=1, \cdots, n$. If we let $C^{\prime}$ be given by $x^{i}=x^{i}(s), i=1, \cdots, h ; x^{i}=0, i>h$, then $C^{\prime}$, while not the projection of $C$ on $G_{h}$ by orthogonal geodesics, will in the cases considered be sufficiently close to $C$ near $P$ to be used for it.

Let $G_{h}$ be the $G_{2}$ determined by $\left.\lambda_{1}\right|^{i}$ and $\left.\lambda_{2}\right|^{i}$. Then at $P$ we have that $d x^{i} / d s$ and $d^{2} x^{i} / d s^{2}$ are in $G_{2}$ and hence equal the corresponding quantities for $C^{\prime}$. By formulas (3) the $\left.\xi_{1}\right|^{i}$ and $\left.\xi_{2}\right|^{i}$ are the same for $C$ and $C^{\prime}$, and hence we have

$$
\left(1 / \rho_{1}\right)=\left(1 / \rho_{1}{ }^{\prime}\right) \text {. }
$$

If $G_{h}$ is $G_{3}$ determined by $\left.\lambda_{1}\right|^{i},\left.\lambda_{2}\right|^{i}$ and $\left.\lambda_{3}\right|^{i}$, it follows as before that $d x^{i} / d s, d^{2} x^{i} / d s^{2}, d^{3} x^{i} / d s^{3}$ are in $G_{3}$ and that they must then equal the corresponding quantities for $C^{\prime}$. Hence it follows that $\left.\xi_{1}\right|^{i},\left.\xi_{2}\right|^{i}$ and $\left.\xi_{3}\right|^{i}$ are the same for the two curves, and that

$$
\left(1 / \rho_{1}\right)=\left(1 / \rho_{1}{ }^{\prime}\right),\left(1 / \rho_{2}\right)=\left(1 / \rho_{2}{ }^{\prime}\right) .
$$

THEOREM 7. If a curve $C$ is projected on its osculating $G_{2}$ or $G_{3}$ at $P$, then the curvatures of $C$ and its projection at $P$ are connected at $P$ by (16) or (17).

We now assume that $G_{h}$, the $h$ th osculating geodesic space of $C$ at $P$, is totally geodesic in $V_{n}$. Then $d^{r} x^{i} / d s^{r}, r \leqq h$, will lie in $G_{h}$, and, since the $d^{r} x^{i} / d s^{r}$ for $C^{\prime}$ are exactly the $G_{h}$ components of $d^{r} x^{i} / d s^{r}$, the two sets will be the same $r \leqq h$. As $\left.\xi_{r}\right|^{i}$ and $\left.\xi_{r}^{\prime}\right|^{i}$ are formed in the same way from the same ordinary derivatives, we have 


$$
\left.\xi_{r}\right|^{i}=\left.\xi_{r}\right|^{i} \text { at } P \text { for } r \leqq h .
$$

It follows from the definition of the curvatures of a curve that if at $P$ the first $h$ associate vectors are known and independent, then the curvatures $\left(1 / \rho_{l}\right), l<h$, and their derivatives $\left(d^{r} / d s^{r}\right)\left(1 / \rho_{s}\right), r+s<h$, of $C$ at $P$ are determined at $P$. From this and the equality of the $\xi_{r} \mid i, r \leqq h$, we have

THEOREM 8. If the first $(h-2)$ curvatures of $C$ at $P$ do not vanish, and if the hth osculating geodesic space $G_{h}$ at $P$ is totally geodesic, then the curvatures $\left(1 / \rho_{l}\right), l<h$, and their derivatives $\left(d^{r} / d s^{r}\right)\left(1 / \rho_{s}\right), r+s<h$, of $C$ at $P$ are equal to the corresponding quantities of the projection of $C$ on $G_{h}$.

LEHIGH UNIVERSITY,

Bethlehem, Pa. 